\title{
Brachial-ankle pulse wave velocity is associated with the risk of osteoporosis: a cross-sectional evidence from a Chinese community-based cohort
}

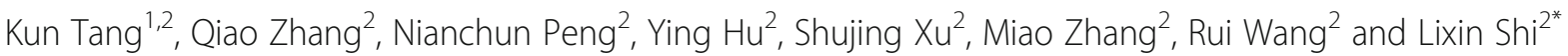

\begin{abstract}
Background: Association of arterial stiffness and osteoporosis has been well documented in elderly population. However, it is not clear whether they co-progress from the early stages through common mechanisms. The object of this study was to evaluate possible associations between arterial stiffness and osteoporosis by measuring brachial-ankle pulse wave velocity (baPWV) and the Osteoporosis Self-Assessment Tool for Asia (OSTA) index among a healthy population of Chinese aged 40 years and older. Whether baPWV can be used as a predictor of osteoporosis on OSTA was further assessed.
\end{abstract}

Methods: This study was cross-sectional in design. Of 3984 adults aged 40 years and older in the Yunyan district of Guiyang (Guizhou, China) who underwent both OSTA and baPWV measurements within 1 month, 1407 were deemed eligible for inclusion (women, 1088; men, 319).

Results: The mean baPWV was $1475 \pm 302 \mathrm{~cm} / \mathrm{s}$ (range,766-3459 cm/s). baPW in 110 individuals with high risk of osteoporosis (OSTA index $<-4)$ was higher than that of individuals with non-high risk $(1733 \pm 461 \mathrm{~cm} / \mathrm{s}$ vs. $1447 \pm$ $304 \mathrm{~cm} / \mathrm{s}, P<0.001)$. OSTA index was negatively correlated with $\operatorname{baPWV}(\rho=-0.296, P<0.001)$ after adjusting for age, sex, body mass index, waist circumference, diastolic blood pressure, and creatinine clearance rate. baPWV was an independent predictor for the presence of high risk of osteoporosis $(\beta=-0.001, P<0.001)$ and was consistent across age and sex subgroups, and the optimal baPWV cutoff value for predicting the presence of high risk of osteoporosis and fracture was $1693 \mathrm{~cm} / \mathrm{s}$. The AUC was 0.722 (95\% confidence interval [Cl], 0.667-0.777; $P<0.001$, sensitivity of $52.8 \%$ and specificity of $83.6 \%$ ).

Conclusions: We conclude that arterial stiffness measured by baPWV is well correlated with the severity of osteoporosis evaluated by OSTA. baPWV index may be a valuable tool for identifying individuals with risk of developing osteoporosis.

Keywords: Brachial-ankle pulse wave velocity (baPWV), Osteoporosis Self-Assessment Tool for Asia (OSTA), Osteoporosis, Arterial stiffness

\footnotetext{
* Correspondence: slx1962@medmail.com.cn

${ }^{2}$ Department of Endocrinology, The Affiliated Hospital of Guizhou Medical University, Guiyang 550000, China

Full list of author information is available at the end of the article
}

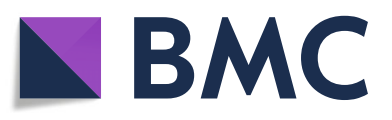

(- The Author(s). 2021 Open Access This article is licensed under a Creative Commons Attribution 4.0 International License, which permits use, sharing, adaptation, distribution and reproduction in any medium or format, as long as you give appropriate credit to the original author(s) and the source, provide a link to the Creative Commons licence, and indicate if changes were made. The images or other third party material in this article are included in the article's Creative Commons licence, unless indicated otherwise in a credit line to the material. If material is not included in the article's Creative Commons licence and your intended use is not permitted by statutory regulation or exceeds the permitted use, you will need to obtain permission directly from the copyright holder. To view a copy of this licence, visit http://creativecommons.org/licenses/by/4.0/ The Creative Commons Public Domain Dedication waiver (http://creativecommons.org/publicdomain/zero/1.0/) applies to the data made available in this article, unless otherwise stated in a credit line to the data. 


\section{Background}

Osteoporosis is a systemic skeletal condition of low bone mass resulting in micro-architectural deterioration of bone tissue, leaving the bones brittle and prone to fractures [1]. According to the latest nationwide, multicenter survey in China, a total of 60 million individuals (10.9 million men and 49.3 million women) are estimated to have osteoporosis [2]. Arterial stiffness, also known as atherosclerosis, is a phenomenon that results with an increase of vascular stiffness loss of elasticity, vessel wall calcification, and blood flow restriction affecting the media of large- and middle-sized arteries [3]. Both arterial stiffness and osteoporosis share some common risk factors and clinical characteristics [4-6].

Decreased bone mineralization (osteoporosis) often occurs concurrently with increased vascular calcification (arterial stiffness), probably due to the common osteogenic and mineralization process shared by bone and vascular cells [7]. Several clinical findings in the past have supported the correlation of osteoporosis or low bone mineral density (BMD) and arterial stiffness, indicating the parallel progression of the two chronic diseases with increased cardiovascular events and higher fracture risk [8-11].

According to the World Health Organization criteria, the gold standard method to assess and diagnose osteoporosis is based on dual-energy X-ray absorptiometry [12]. However, this tool also has certain limitations (e.g., high costs and large size of the equipment, exposure to ionizing radiation) that limit its widespread application for population screenings. On the other hand, some elderly and high-risk individuals often do not undergo regular screening and comprehensive examinations, probably due to health or financial reasons [13]. Among several currently used tools [14-16], the Osteoporosis SelfAssessment Tool for Asians (OSTA) score developed by WHO has been shown to be the simplest and highly effective tool to identify both women and men at risk for osteoporosis [6, 17]. It has been concluded that the OSTA was a useful screening tool to detect osteoporosis in middle-aged and old women in the Chengdu region of China [18]. A significant correlation in a positive direction was found between the OSTA index and BMD ( $T$ score) measured by dual-energy X-ray absorptiometry $[19,20]$. Brachial-ankle pulse wave velocity (baPWV), recording the time taken by the pressure wave to travel over a specific distance, is the most common measure of arterial stiffness in Asian populations and is widely used as it is convenient, noninvasive, and inexpensive [21-23].

The measurement of arterial stiffness of healthy adults undergoing screening medical examination might help to make decisions on early intervention for osteoporosis or facture risk, since correlation of arterial stiffness by measuring baPWV and osteoporosis (BMD or other assessment indices) has been reported in recent studies $[24,25]$. Xuan et al.'s work was the first to investigate the correlation between arterial stiffness (baPWV) and OSTA index in Chinese population. However, their findings were based on 129 elderly Chinese communitydwelling individuals with mean age above 83.2; it is not clear whether the one was the result of the other or they co-progress from the early stages through common mechanisms. Therefore, the aim of this study was (1) to investigate the relation between baPWV measurement and the OSTA index and (2) to evaluate baPWV as a predictor of risk of osteoporosis in 3984 Chinese healthy adults aged 40 years and older.

\section{Methods}

\section{Study population}

The present study was part of the baseline survey for the Risk Evaluation of Cancers in Chinese Diabetic Individuals: a longitudinal (REACTION) study, which was conducted among 259,657 adults aged 40 years and older in 25 communities across mainland China from 2011 to 2012 [26-29]. The study protocol was approved by the ethics committees at Guizhou Medical University Affiliated Hospital and informed consent had been obtained from each participant who signed a form before the study. A total of 3984 adults aged 40 to 80 years old who lived 5 years or longer in the Yunyan district of Guiyang (Guizhou, China) were selected by cluster random sampling. Major exclusion criteria are shown in Fig. 1 and 1407 individuals were deemed eligible for inclusion (women, 1088; men, 319).

\section{Study method}

(1) Questionnaire survey: Information collected includes demographic and other background information (sex, age, occupation, home address, contact number, family history, marital and childbirth history, etc.) and past medical history (history of diabetes, hypertension, dyslipidemia, cardiovascular disease history, etc. and treatment of related diseases).

(2) Physical examination: Height, body weight, waist circumference (WC), and blood pressure (BP) were measured. BP was measured three times in the supine position and the average was taken as the measurement value. The body mass index (BMI = weight/height ${ }^{2}$ ) was calculated.

(3) Sample collection and measurement: Venous blood samples were obtained in the morning and an overnight fasting. Fasting plasma glucose (FPG) concentration and postprandial glucose (PPG) levels were measured using the glucose oxidase technique with a Roche Hitachi P800 autoanalyzer (Roche 


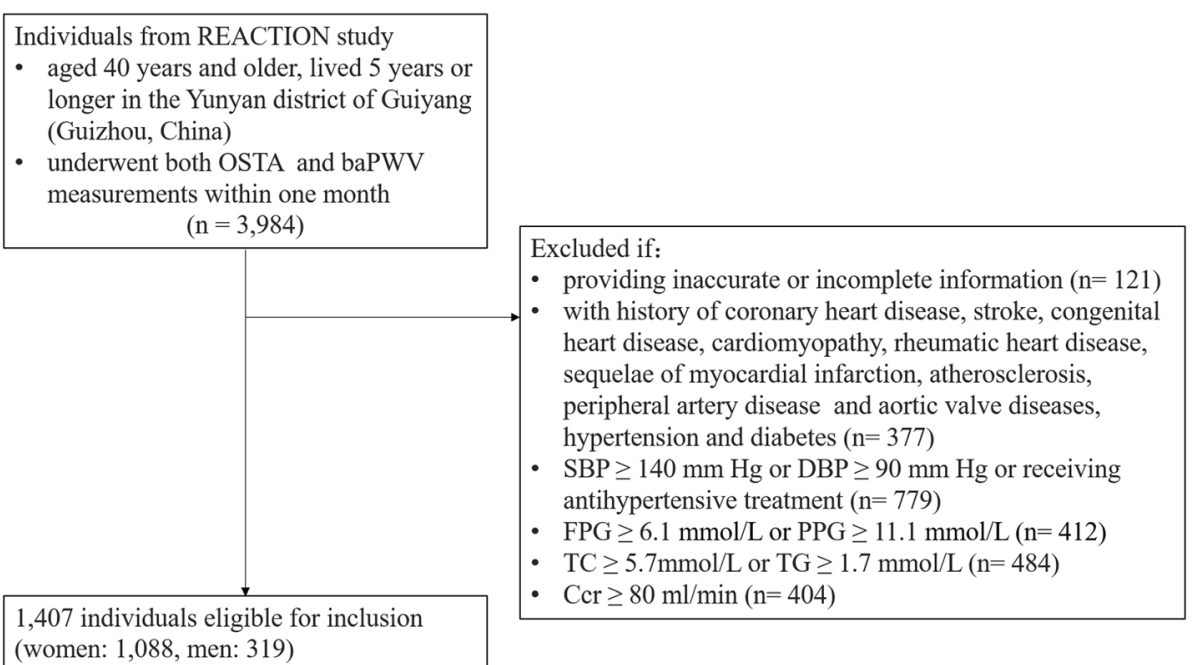

Fig. 1 Workflow of sample enrollment of this study

Diagnostics GmbH, Mannheim). Lipid panel test including total cholesterol (TC), high-density lipoprotein cholesterol (HDL), low-density lipoprotein cholesterol (LDL), and triglyceride (TG) was measured using an autoanalyzer (ARCHITECT Ci16200). We used creatinine clearance rate (Ccr) as an indicator of kidney function.

(4) baPWV measurement: After a 10-min rest in the supine position, baPWV was automatically measured using the Omron device (BP-203RPEIII VP-1000 device; Omron Health Care, Kyoto, Japan) by a single observer with high level of operator experience. The validity and reliability of this device has been previously reported [30]. Cuffs with sensors were wrapped on both upper arms and ankles. The transmission times and distances between the cuffs of arms and legs were recorded, and the device was able to compute baPWV automatically as the ratio of the travel distance divided by the time difference between the pulse waves. We used the mean value of the right and left baPWVs in our analysis [31, 32].

(5) OSTA index calculation: OSTA index was calculated using the formula of (weight in kilograms - age in years) $\times 0.2$, established by Koh et al. (2001) [17]. An OSTA index of -1 to -4 is regarded as medium risk of sustaining osteoporosis, greater than -1 as low risk, and less than -4 as high risk [33, 34].

(6) Subgroup stratification: The study population was then divided into subgroups according to sex and age ( $<65$ vs. $\geq 65$ years old) for analysis, considering osteoporosis starts earlier and gets worse faster in women; however, starting at about age 65 , both sexes lose bone at about the same rate $[35,36]$.

\section{Statistical analyses}

Continuous variables were expressed as means \pm standard deviation (SD). For assessment of the differences in a variable between 2 groups, the unpaired $t$ test was applied. Spearman's rank correlation analyses and partial correlation analyses were conducted to study the associations between baPWV and OSTA, anthropometric indices, and serum biochemical parameters. Multivariable regression analysis was performed to assess whether baPWV was independently associated with osteoporosis and using variables showing significant relationships with OSTA in Spearman's rank correlation analysis. To explore the best cutoff value of baPWV for predicting high risk of osteoporosis and fracture (OSTA $\leq-4)$, receiver operating characteristic (ROC) curve analysis was used. The predictive accuracy was presently with the area under the curve (AUC). Youden index, which was sensitivity + specificity -1 , was calculated to enable the selection of an optimal threshold value (cutoff point) [37]. The statistical analysis was performed using SPSS 19.0 software (SPSS, Inc., Chicago, IL, USA).

\section{Results}

\section{Baseline characteristics}

The baseline anthropometric parameters and biochemical indices from 1407 individuals are shown in Table 1. Males (age, $62.33 \pm 7.99$ years) were significantly older than females (age, $58.14 \pm 7.52$ years). Females had higher HDL, TC, and Ccr $(P<0.05)$. BMI, SBP, DBP, and PPG were higher in men than in women (all $P<$ 0.05). For total individuals, the mean baPWV was 1475 
Table 1 General characteristics of male and female patients

\begin{tabular}{lllll}
\hline $\boldsymbol{N}$ & Male & Female & $\boldsymbol{T}$ value & $\boldsymbol{P}$ value \\
& $\mathbf{3 1 9}$ & $\mathbf{1 0 8 8}$ & & \\
\hline Age (year) & $62.33 \pm 7.99$ & $58.14 \pm 7.52$ & 8.347 & $<0.001$ \\
BMI $\left(\mathrm{kg} / \mathrm{m}^{2}\right)$ & $23.04 \pm 2.74$ & $22.54 \pm 2.89$ & 2.789 & 0.005 \\
WC $(\mathrm{cm})$ & $85.14 \pm 7.72$ & $84.16 \pm 8.57$ & 1.832 & 0.067 \\
SBP $(\mathrm{mmHg})$ & $118.89 \pm 11.23$ & $113.77 \pm 12.37$ & 6.648 & $<0.001$ \\
DBP $(\mathrm{mmHg})$ & $76.22 \pm 7.66$ & $73.00 \pm 7.99$ & 6.401 & $<0.001$ \\
FPG (mmol/L) & $5.28 \pm 0.47$ & $5.26 \pm 0.40$ & 0.513 & 0.608 \\
PPG (mmol/L) & $7.02 \pm 1.62$ & $6.79 \pm 1.52$ & 2.276 & 0.023 \\
HDL (mmol/L) & $1.44 \pm 0.28$ & $1.49 \pm 0.28$ & 2.440 & 0.015 \\
LDL (mmol/L) & $2.73 \pm 0.57$ & $2.78 \pm 0.52$ & 1.450 & 0.147 \\
TC (mmol/L) & $4.72 \pm 0.62$ & $4.82 \pm 0.58$ & 2.472 & 0.014 \\
TG (mmol/L) & $1.14 \pm 0.30$ & $1.11 \pm 0.31$ & 1.508 & 0.132 \\
Ccr (mL/min) & $89.23 \pm 8.77$ & $92.68 \pm 11.13$ & 5.789 & $<0.001$ \\
baPWV (cm/s) & $1461.43 \pm 391.11$ & $1471.55 \pm 307.43$ & 0.425 & 0.671 \\
OSTA & $0.34 \pm 2.53$ & $-0.73 \pm 2.30$ & 6.813 & $<0.001$ \\
\hline
\end{tabular}

$B M I$ body mass index, WC waist circumstances, $S B P$, systolic blood pressure, $D B P$ diastolic blood pressure, $F P G$ fasting plasma glucose, $P P G$ postprandial glucose, $H D L$ high-density lipoprotein cholesterol, $L D L$ low-density lipoprotein cholesterol, TC total cholesterol, TG triglyceride, C Cr creatinine clearance, baPWV brachial-ankle pulse wave velocity, OSTA Osteoporosis Self-Assessment Tool for Asia

$\pm 302 \mathrm{~cm} / \mathrm{s}$ (range, $766-3,459 \mathrm{~cm} / \mathrm{s}$ ) and there was no significant difference between males and females in baPWV. Risk of osteoporosis measured by OSTA index is significantly higher in females $(0.34 \pm 2.53$ in males vs. $-0.73 \pm 2.30$ in females).

\section{Association of OSTA with baPWV and biochemical parameters}

Spearman's rank correlation analysis presented a significant negative correlation between OSTA index and baPWV $(\rho=-0.290, \mathrm{P}<0.001$ ) (Table 2; Fig. 2). Further, we calculated the partial correlation between OSTA index and baPWV, adjusting for age, sex, BMI, WC, DBP, and Ccr. The correlation in a negative direction between OSTA index and baPWV was not significantly affected by other demographic or biochemical variables $(\rho=-0.294, P<0.001)$.

\section{baPWV as a predictor of high risk of osteoporosis}

Stepwise multiple regression analysis was further applied to access the independent relationships between OSTA and baPWV. As shown in Table 3, multivariate stepwise regression analysis revealed that among those factors that showed associations with OSTA in correlation analysis, baPWV was an independent factor responsible for the changes in OSTA $(\beta=-0.001, P<0.001)$. The regression equation was OSTA $=5.317+0.279 \times \mathrm{BMI}-$ $0.182 \times$ Age $-1.642 \times$ Sex $+0.042 \times \mathrm{WC}-0.001 \times$ baPWV.
Table 2 Correlations of OSTA index with baPW and various clinical and biochemical parameters

\begin{tabular}{lll}
\hline & OSTA index & \\
\cline { 2 - 3 } & $\boldsymbol{\rho}$ & $\boldsymbol{P}$ value \\
\hline baPW & -0.290 & $<0.001$ \\
Age & -0.543 & $<0.001$ \\
Sex & -0.174 & $<0.001$ \\
BMl & 0.459 & $<0.001$ \\
WC & 0.380 & $<0.001$ \\
FPG & 0.000 & 0.993 \\
PPG & -0.036 & 0.172 \\
SBP & 0.000 & 0.996 \\
DBP & 0.182 & $<0.001$ \\
CCr & 0.239 & $<0.001$ \\
HDL & -0.042 & 0.113 \\
LDL & 0.009 & 0.724 \\
TC & -0.022 & 0.402 \\
TG & 0.024 & 0.370
\end{tabular}

$B M I$ body mass index, WC waist circumstances, $S B P$ systolic blood pressure, $D B P$ diastolic blood pressure, $F P G$ fasting plasma glucose, $P P G$ postprandial glucose, $H D L$ high-density lipoprotein cholesterol, $L D L$ low-density lipoprotein cholesterol, TC total cholesterol, TG triglyceride, C Cr creatinine clearance, baPWV brachial-ankle pulse wave velocity, OSTA Osteoporosis Self-Assessment Tool for Asia

Overall, 110 individuals with high risk of osteoporosis (OSTA index < - 4) was identified; baPWV measurement of these individuals was higher than that of individuals with non-high risk $(1733 \pm 461 \mathrm{~cm} / \mathrm{s}$ vs. $1447 \pm$ $304 \mathrm{~cm} / \mathrm{s}, P<0.001)$. In the ROC curve analysis, the AUC was 0.722 (95\% confidence interval [CI], 0.667$0.777 ; P<0.001$ ), and the optimal cutoff point of baPWV value for predicting the presence of high risk of

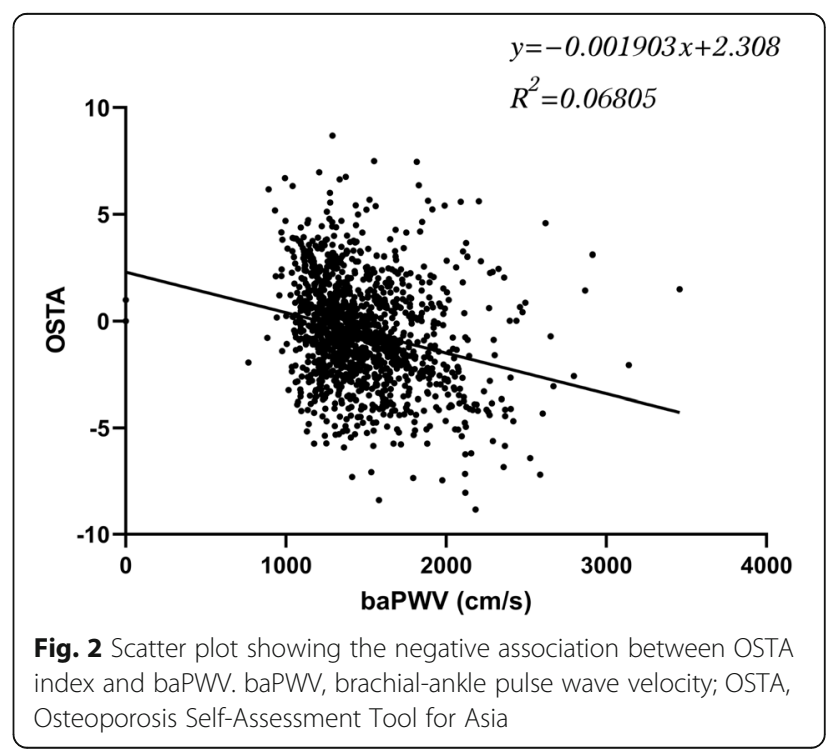


Table 3 Multivariate stepwise regression analysis showing the factors independently associated with OSTA

\begin{tabular}{lllll}
\hline Characteristics & $\boldsymbol{\beta}$ & SE & Standardized $\boldsymbol{\beta}$ & $\boldsymbol{P}$ \\
\hline BMl & 0.279 & 0.021 & 0.334 & $<0.001$ \\
Age & -0.182 & 0.005 & -0.594 & $<0.001$ \\
Sex & -1.642 & 0.093 & -0.287 & $<0.001$ \\
WC & 0.042 & 0.007 & 0.146 & $<0.001$ \\
baPWV & -0.001 & 0.000 & -0.185 & $<0.001$ \\
\hline
\end{tabular}

Note: Multivariable stepwise regression analysis was performed using variables showing significant relationships with OSTA in Spearman's rank correlation analysis

$B M I$ body mass index, WC waist circumstances, $C \mathrm{Cr}$ creatinine clearance, baPWV brachial-ankle pulse wave velocity, OSTA Osteoporosis Self-Assessment Tool for Asia

osteoporosis and fracture was $1693 \mathrm{~cm} / \mathrm{s}$ with sensitivity of $52.8 \%$ and specificity of $83.6 \%$ (Supplementary file 1 ).

\section{Age and sex subgroup analysis}

Further subgroup analysis showed that baPWV was an independent factor responsible for the changes in OSTA in each subgroup (Table 4). In male population, the AUC was 0.716 (95\% confidence interval [CI], 0.5450.886; $P=0.005$ ), slightly lower than the AUC in female population (AUC 0.726; 95\% confidence interval [CI], 0.669-0.783; $P<0.001)$. In individuals with age under 65, the AUC was 0.789 (95\% confidence interval [CI], 0.650 to $0.929 ; P<0.001$ ), much higher than the AUC in individuals with age above 65 (AUC 0.662; 95\% confidence interval $[\mathrm{CI}], 0.595$ to $0.729 ; P<0.001$ ) (Fig. 2).

\section{Discussion}

The present study, conducted in a large Chinese community-based cohort, showed that baPWV, a promising yet relatively simple test, has a significant negative correlation with the degree of risk for osteoporosis as quantified by OSTA. The association between baPWV and osteoporosis risk was independent of age, sex, and traditional risk factors, which was concluded using the multivariable analysis. For predicting high risk of osteoporosis and fracture, we showed a baPWV cutoff value of $1693 \mathrm{~cm} / \mathrm{s}$ that had the best predictive power resulted in AUCs of about 0.722 .

Correlations between arterial stiffness and BMD have been frequently reported in both retrospective and cross-sectional studies. A cross-sectional study involving around four thousand Chinese men and women aged 65-92 reported that ankle-brachial index (ABI) as a measurement for peripheral arteriosclerosis was positively correlated with hip BMDs [38]. Further, prospective studies have also reported findings in evaluating whether low BMD predicts cardiovascular events. In China, a prospective osteoporosis study followed 1724 postmenopausal women for 5 years; they found the presence of aortic calcifications assessed using semiquantitative radiography at baseline was associated with a higher rate of vertebral fractures $(12.2 \%$ vs. $4.5 \%$ in women with and without aortic calcifications $(P=0.01)$ [39]. This study was pooled in a further meta-analysis on the relationship of aortic calcifications to the risk of fracture, which demonstrated that aortic calcifications were

Table 4 Multivariate stepwise regression analysis showing the factors independently associated with OSTA in age and sex subgroups

\begin{tabular}{|c|c|c|c|c|c|}
\hline & Characteristics & $\beta$ & SE & Standardized $\beta$ & $P$ \\
\hline \multirow[t]{4}{*}{ Male population } & Age & -0.170 & 0.013 & -0.535 & $<0.001$ \\
\hline & WC & 0.130 & 0.013 & 0.396 & $<0.001$ \\
\hline & baPWV & -0.001 & 0.000 & -0.195 & $<0.001$ \\
\hline & $\mathrm{Ccr}$ & -0.013 & 0.006 & -0.086 & 0.042 \\
\hline \multirow[t]{5}{*}{ Female population } & Age & -0.187 & 0.005 & -0.612 & $<0.001$ \\
\hline & WC & 0.035 & 0.007 & 0.130 & $<0.001$ \\
\hline & bapW & -0.001 & 0.000 & -0.190 & $<0.001$ \\
\hline & BMI & 0.300 & 0.021 & 0.379 & $<0.001$ \\
\hline & DBP & 0.011 & 0.005 & 0.038 & 0.033 \\
\hline \multirow[t]{3}{*}{ Ages $<65$ population } & baPWV & -0.001 & 0.000 & -0.190 & $<0.001$ \\
\hline & BMI & 0.400 & 0.018 & 0.540 & $<0.001$ \\
\hline & Sex & -1.139 & 0.134 & -0.204 & $<0.001$ \\
\hline \multirow[t]{3}{*}{ Ages $65+$ population } & bapW & -0.002 & 0.000 & -0.304 & $<0.001$ \\
\hline & WC & 0.120 & 0.011 & 0.440 & $<0.001$ \\
\hline & Sex & -2.169 & 0.193 & -0.451 & $<0.001$ \\
\hline
\end{tabular}

Note: Multivariable stepwise regression analysis was performed using variables showing significant relationships with OSTA in Spearman's rank correlation analysis $B M I$ body mass index, WC waist circumstances, $C c r$ creatinine clearance, baPWV brachial-ankle pulse wave velocity, OSTA Osteoporosis Self-Assessment Tool for Asia, DBP diastolic blood pressure, $H D L$ high-density lipoprotein cholesterol 
significantly and independently associated with a higher fracture risk, recruiting 14,632 participants in total [38]. For peripheral arterial disease (PAD), in a prospective study of 1332 individuals, peripheral arterial disease (PAD) measured by ABI was not associated with the occurrence of fractures [40]. However, another study involved measurements of $\mathrm{ABI}$ and of BMD in 5781 men aged 65 years or older found inconsistent results with the previous mentioned study, which reported that individuals with PAD had higher rates of bone loss and increased risk of non-spine fractures [41].

The possible pathophysiological mechanism linking high baPWV and osteoporosis could be oxidative stress, an imbalance between exposure to toxic reactive oxygen species (ROS) and antioxidant systems, considering that it is associated with both arterial stiffness and osteoporosis [42, 43]. Hormonal changes associated with menopause and aging affect both arterial stiffness and bone resorption and reconstruction; it could be another mechanism behind the predictive value of baPWV for osteoporosis risk [44]. Diverse studies revealed significant correlations between the severity of arterial stiffness/osteoporosis and inflammatory markers; it could also be another common pathway for the pathogenesis of arterial stiffness and osteoporosis [45]. Since in population ages 40 to 65 , we found the highest predictive value of baPWV in individuals at high risk of osteoporosis, proposing arterial stiffness and osteoporosis might co-progress from the early stages of bone loss through common mechanisms.

It is important to obtain a simple and effective way to predict osteoporosis and cardiovascular disease risk, considering the huge aging population of the People's Republic of China. As far as we know, the first study to report the relationship between OSTA and baPWV in Chinese population was published in 2019, involving 129 elderly individuals and suggests the OSTA index was negatively correlated with baPWV in linear regression analysis and baPWV as a valuable predictive factor for potential osteoporotic risk [46]. Our findings have proven the association in a large Chinese communitybased cohort involving 1407 individuals; the AUC was 0.722 in ROC curve analysis for evaluating baPWV as a predictor of high risk of osteoporosis and fracture (OSTA $\leq-4)$, close to the AUC (0.742) reported in the abovementioned study [46]. However, we reported a low sensitivity value of $51.8 \%$ for the optimal cutoff point of baPWV value in predicting the presence of high risk of osteoporosis and fracture. This is probably due to the age heterogeneity in our study, since we recruited all the adults aged 40 years and older (Fig. 3).

Low BMI having been frequently shown to increase risk of fracture, possibly due to its association with bone loss, less soft tissue, and muscle weakness [47]. In our

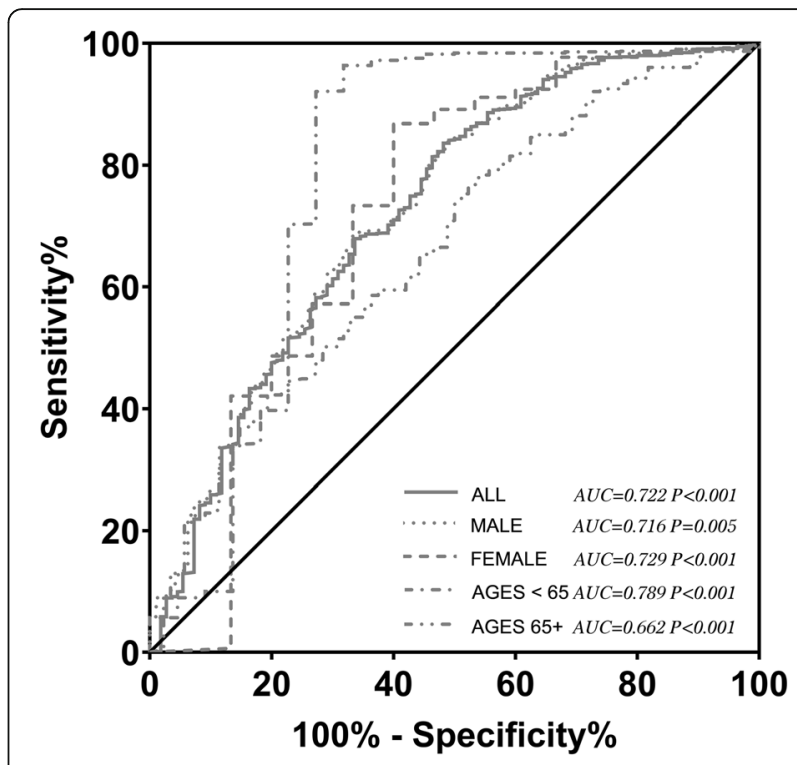

Fig. 3 ROC curve of the association between baPWV and the high risk of osteoporosis and fracture (corresponding to OSTA $<-4.0$ ) in total sample and subgroups. ROC, receiver operating characteristic; baPWW, brachial-ankle pulse wave velocity; OSTA: Osteoporosis SelfAssessment Tool for Asia; AUC, area under curve; $\mathrm{Cl}$, confidence interval

study, we found BMI and WC are positively associated with OSTA. However, there were studies reporting high BMI increases the risk of osteoporotic and hip fractures risk [48]. In a meta-analysis involving 398,610 women, it is interesting to find that the association between BMI and fracture risk is complex, which differs across skeletal sites. Since there is no consistent conclusion from these analyses, a healthy and normal BMI might be suggested to help minimize the risk of fracture risk. Only few observational studies have investigated the association between serum lipid level and bone fractures in Chinese population, and the conclusions between studies are also controversial. In our study, we found a negative association between HDL and OSTA. In a cross-sectional study involving around 5000 healthy volunteers, the authors reported that the subjects with a BMI lower than18.5 had a higher incidence of osteoporosis than BMI $\geq 18.5$ in both sexes [49]. Another cross-sectional study including 1791 participants $(62.1 \%$ postmenopausal women and 213 fractures) reported a significant positive association between HDL-C level and risk of osteoporotic fracture in total participants (OR 1.37, $P=$ 0.023). These findings are consistent with what have been reported in our study. However, a recent study applied two-sample Mendelian randomization (MR) methods to explore the causal association between blood lipid levels and fracture. They reported that HDL may have an indirect influence on fracture mediated by BMD [50]. According to what we have discussed above, the 
relationship between lipid levels in the blood and the risk of fracture is currently controversial and the causal association remains elusive; further research is required.

The study described here included 1407 participants aged 40 years and older. This relatively large sample size and subgroup analysis strengthens the thoroughness of our findings. According to what we have found, we proposed that baPWV had moderate discrimination ability for high risk of osteoporosis, especially for population ages 40 to 65 . However, several limitations of our study should be thoroughly discussed. First, as a crosssectional study, no causal inference can be concluded. Further well-designed longitudinal studies are needed to validate the relationship identified in this study. Second, OSTA was the only measurement representing osteoporosis and fracture risk in this study. Future studies should search a link between other markers of osteoporosis and arterial stiffness, such as Mandibular cortical width, a marker of osteoporosis detected by dental panoramic radiographs [51]. Third, information such as physical activity and alcohol consumption was not collected. These behavioral factors might have to affect fracture risk profoundly.

\section{Conclusion}

In summary, we have found the independent predictive value of baPWV for osteoporosis risk in a large Chinese community-based cohort. Furthermore, the inverse association of the OSTA index and baPWV was statistically significant. baPWV may be a simple and useful indicator of osteoporosis and fracture risk.

\section{Supplementary Information}

The online version contains supplementary material available at https://doi. org/10.1186/s13018-020-02125-3.

Additional file 1: Table S1. Youdem index for the ROC curve analysis.

\section{Abbreviations \\ baPWV: Brachial-ankle pulse wave velocity; OSTA: Osteoporosis Self- Assessment Tool for Asia; BMD: Bone mineral density; ROC: Receiver operating characteristic; AUC: Area under the curve; SD: Standard deviation; PAD: Peripheral arterial disease; ROS: Reactive oxygen species; BMl: Body mass index; WC: Waist circumstances; SBP: Systolic blood pressure; DBP: Diastolic blood pressure; FPG: Fasting plasma glucose; PPG: Postprandial glucose; HDL: High-density lipoprotein cholesterol; LDL: Low-density lipoprotein cholesterol; TC: Total cholesterol; TG: Triglyceride; Ccr: Creatinine clearance}

\section{Acknowledgements}

The authors would like to thank all participants in this study.

\section{Authors' contributions}

Conceptualization: KT and LXS. Data collection and data curation: QZ, NCP, YH. Methodology: SJX, MZ, RW. Writing - original draft: KT. Writing - review and editing and supervision: LXS. Funding acquisition: LXS. The authors read and approved the final manuscript.

\section{Funding}

This work was supported by the Ministry of Science and Technology under Grants (NO: 2016YFC0901203) and 201502007 from the Ministry of Health. This work was supported by the National Key Research and Development Program of China (NO: 2016YFC0901203) and 201502007 from the Ministry of Health.

\section{Availability of data and materials}

The datasets used and/or analyzed during the current study are available from the corresponding author on reasonable request.

\section{Ethics approval and consent to participate}

The present study was part of the baseline survey for the Risk Evaluation of Cancers in Chinese Diabetic Individuals: a longitudinal (REACTION) study. The study protocol was approved by the ethics committees at Guizhou Medical University Affiliated Hospital and informed consent had been obtained from each participant who signed a form before the study.

\section{Consent for publication}

Written informed consent for publication was obtained from all participants.

\section{Competing interests}

The authors declare that they have no competing interests

\section{Author details}

'Department of Endocrinology, The Second Affiliated Hospital of Soochow University, Suzhou 215004, China. ${ }^{2}$ Department of Endocrinology, The Affiliated Hospital of Guizhou Medical University, Guiyang 550000, China.

Received: 19 September 2020 Accepted: 27 November 2020

Published online: 04 January 2021

\section{References}

1. Osterhoff G, Morgan EF, Shefelbine SJ, Karim L, McNamara LM, Augat P. Bone mechanical properties and changes with osteoporosis. Injury. 2016; 47(Suppl 2):S11-20.

2. Zeng Q, Li N, Wang Q, Feng J, Sun D, Zhang Q, Huang J, Wen Q, Hu R, Wang L, Ma Y, Fu X, Dong S, Cheng X. The prevalence of osteoporosis in China, a nationwide, multicenter DXA survey. J Bone Miner Res. 2019;34(10):1789-97.

3. Avramovski P, Avramovska M, Sikole A. Bone strength and arterial stiffness impact on cardiovascular mortality in a general population. J Osteoporosis. 2016;2016:7030272.

4. McFarlane SI, Muniyappa R, Shin JJ, Bahtiyar G, Sowers JR. Osteoporosis and cardiovascular disease. Endocrine. 2004;23(1):1-10.

5. Tankó LB, Christiansen C, Cox DA, Geiger MJ, McNabb MA, Cummings SR. Relationship between osteoporosis and cardiovascular disease in postmenopausal women. J Bone Mineral Res. 2005:20(11):1912-20.

6. Crepaldi G, Maggi S. Epidemiologic link between osteoporosis and cardiovascular disease. J Endocrinological Investigation. 2009;32(4 Suppl):2-5.

7. Sage AP, Tintut Y, Demer LL. Regulatory mechanisms in vascular calcification. Nat Rev Cardiol. 2010;7(9):528-36.

8. Mikumo M, Okano H, Yoshikata R, Ishitani K, Ohta H. Association between lumbar bone mineral density and vascular stiffness as assessed by pulse wave velocity in postmenopausal women. J Bone Miner Metab. 2009;27(1): 89-94.

9. Chen $Y$, Zhao $X$, Wu H. Arterial Stiffness: A focus on vascular calcification and its link to bone mineralization. Arteriosclerosis Thrombosis Vascular Biol. 2020:40(5):1078-93.

10. Charitaki E, Davenport A. Aortic pulse wave velocity in haemodialysis patients is associated with the prescription of active vitamin $D$ analogues. J Nephrol. 2014;27(4):431-7.

11. Stojanovic Ol, Lazovic M, Lazovic M, Vuceljic M. Association between atherosclerosis and osteoporosis, the role of vitamin D. Arch Med Sci. 2011; 7(2):179-88

12. Ukon Y, Makino T, Kodama J, Tsukazaki H, Tateiwa D, Yoshikawa H, et al. Molecular-based treatment strategies for osteoporosis: a literature review. Int J Mol Sci. 2019;20(10):2557.

13. Pisani P, Renna MD, Conversano F, Casciaro E, Muratore M, Quarta E, et al. Screening and early diagnosis of osteoporosis through $\mathrm{X}$-ray and ultrasound based techniques. World J Radiol. 2013;5(11):398-410. 
14. Lydick E, Cook K, Turpin J, Melton M, Stine R, Byrnes C. Development and validation of a simple questionnaire to facilitate identification of women likely to have low bone density. Am J Managed Care. 1998;4(1):37-48.

15. Cadarette SM, Jaglal SB, Kreiger N, Mclsaac WJ, Darlington GA, Tu JV. Development and validation of the Osteoporosis Risk Assessment Instrument to facilitate selection of women for bone densitometry. CMAJ. 2000;162(9):1289-94

16. Sedrine WB, Chevallier T, Zegels B, Kvasz A, Micheletti MC, Gelas B, et al. Development and assessment of the Osteoporosis Index of Risk (OSIRIS) to facilitate selection of women for bone densitometry. Gynecological Endocrinol. 2002;16(3):245-50.

17. Koh LK, Sedrine WB, Torralba TP, Kung A, Fujiwara S, Chan SP, et al. A simple tool to identify asian women at increased risk of osteoporosis. Osteoporosis Int. 2001;12(8):699-705.

18. Huang JY, Song WZ, Zeng HR, Huang M, Wen QF. Performance of the Osteoporosis Self-Assessment Tool for Asians (OSTA) in screening osteoporosis among middle-aged and old women in the Chengdu Region of China. J Clin Densitometry. 2015;18(4):539-45.

19. Yang $Y$, Wang B, Fei Q, Meng Q, Li D, Tang H, et al. Validation of an osteoporosis self-assessment tool to identify primary osteoporosis and new osteoporotic vertebral fractures in postmenopausal Chinese women in Beijing. BMC Musculoskeletal Disord. 2013;14:271

20. Machado P, Coutinho M, da Silva JA. Selecting men for bone densitometry: performance of osteoporosis risk assessment tools in Portuguese men. Osteoporosis Int. 2010;21(6):977-83.

21. Nakamura M, Yamashita T, Yajima J, Oikawa Y, Sagara K, Koike A, et al. Brachial-ankle pulse wave velocity as a risk stratification index for the shortterm prognosis of type 2 diabetic patients with coronary artery disease. Hypertension Res. 2010;33(10):1018-24.

22. Maeda Y, Inoguchi T, Etoh E, Kodama Y, Sasaki S, Sonoda N, et al. Brachialankle pulse wave velocity predicts all-cause mortality and cardiovascular events in patients with diabetes: the Kyushu prevention study of atherosclerosis. Diabetes care. 2014;37(8):2383-90.

23. Pearson TA. New tools for coronary risk assessment: what are their advantages and limitations? Circulation. 2002;105(7):886-92.

24. Kim N-L, Suh H-S. Correlation of arterial stiffness and bone mineral density by measuring brachial-ankle pulse wave velocity in healthy Korean women. Korean J Fam Med. 2015:36(6):323-7.

25. K-i H, Tomiyama H, Okazaki R, Arai T, Koji Y, Zaydun G, et al. Increased pulse wave velocity associated with reduced calcaneal quantitative osteo-sono index: possible relationship between atherosclerosis and osteopenia. J Clin Endocrinol Metab. 2003;88:2573-8.

26. Ning G, Group RS. Risk Evaluation of cAncers in Chinese diabeTic Individuals: a IONgitudinal (REACTION) study. J diabetes. 2012;4(2):172-3.

27. Bi Y, Lu J, Wang W, Mu Y, Zhao J, Liu C, et al. Cohort profile: Risk evaluation of cancers in $C$ hinese diabetic individuals: a longitudinal (REACTION) study (队列简介: 中国糖尿病患者肿瘤发生风险的纵向研究 (REACTION 研究)). J diabetes. 2014;6(2):147-57.

28. Lu J, Bi Y, Wang T, Wang W, Mu Y, Zhao J, et al. The relationship between insulin-sensitive obesity and cardiovascular diseases in a Chinese population: results of the REACTION study. Int J Cardiol. 2014;172(2):388-94.

29. Ning G, Bloomgarden Z. Diabetes and cancer: Findings from the REACTION study 糖尿病与恶性肿瘤: 来自 REACTION 研究的发现. J Diabetes. 2015; 7(2):143-4.

30. Komine H, Asai Y, Yokoi T, Yoshizawa M. Non-invasive assessment of arterial stiffness using oscillometric blood pressure measurement. Biomed Eng Online. 2012:11:6

31. Zhu, Chen, Lu. Association between the accumulation of metabolic syndrome abnormal components and arterial pulse wave velocity among adult individuals undergoing routine health examination. Zhonghua xin xue guan bing za zhi. 2018;2(3):33-35.

32. Yamashina A, Tomiyama H, Takeda K, Tsuda H, Arai T, Hirose K, et al. Validity, reproducibility, and clinical significance of noninvasive brachial-ankle pulse wave velocity measurement. Hypertension Res. 2002;25(3):359-64.

33. Rau CS, Kuo PJ, Wu SC, Chen YC, Hsieh HY, Hsieh CH. Association between the Osteoporosis Self-Assessment Tool for Asians Score and Mortality in Patients with Isolated Moderate and Severe Traumatic Brain Injury: A Propensity ScoreMatched Analysis. Int J Environ Res Public Health. 2016;13(12):1203.

34. Zhang HM, Liu HL, Wang X, Chen W, Chen D, Zhang ZZ, Wang HM. Clinical value of self-assessment risk of osteoporosis in Chinese. Open Med (Wars). 2016;11(1):190-95
35. Stephen AB, Wallace WA. The management of osteoporosis. The Journal of bone and joint surgery British volume. 2001;83(3):316-23.

36. Herrera A, Lobo-Escolar A, Mateo J, Gil J, Ibarz E, Gracia L. Male osteoporosis: a review. World J Orthop. 2012;3(12):223-34.

37. Schisterman EF, Perkins NJ, Liu A, Bondell H. Optimal cut-point and its corresponding Youden Index to discriminate individuals using pooled blood samples. Epidemiology (Cambridge, Mass). 2005;16(1):73-81.

38. Wei D, Zheng G, Gao Y, Guo J, Zhang T. Abdominal aortic calcification and the risk of bone fractures: a meta-analysis of prospective cohort studies. J Bone Mineral Metabol. 2018;36(4):439-46.

39. Zhou R, Zhou H, Cui M, Chen L, Xu J. The Association between aortic calcification and fracture risk in postmenopausal women in China: the Prospective Chongqing Osteoporosis Study. PLOS ONE. 2014;9(5):e93882.

40. von Mühlen D, Allison M, Jassal SK, Barrett-Connor E. Peripheral arterial disease and osteoporosis in older adults: the Rancho Bernardo Study. Osteoporosis International. 2009;20(12):2071-8.

41. Collins T, Ewing S, Diem S, Taylor B, Orwoll E, Cummings S, et al. Peripheral arterial disease is associated with higher rates of hip bone loss and increased fracture risk in older men. Circulation. 2009;119:2305-12.

42. Mozos I, Luca CT. Crosstalk between oxidative and nitrosative stress and arterial stiffness. Current vascular pharmacology. 2017;15(5):446-56.

43. Zhou Q, Zhu L, Zhang D, Li N, Li Q, Dai P, Mao Y, Li X, Ma J, Huang S. Oxidative stress-related biomarkers in postmenopausal osteoporosis: a systematic review and meta-analyses. Dis Markers. 2016;2016:7067984. https://doi.org/10.1155/2016/7067984

44. Zhang M, Bai L, Kang J, Ge J, Peng W. Links between arterial stiffness and bone mineral density in middle-aged and elderly Chinese individuals: a cross-sectional study. BMJ open. 2019;9(8):e029946.

45. Mozos I, Malainer C, Horbańczuk J, Gug C, Stoian D, Luca CT, et al. Inflammatory markers for arterial stiffness in cardiovascular diseases. Front Immunol. 2017;8:1058

46. Xuan Y, Wang W, Zhang H, Tan I, Butlin M. Avolio A, et al. Osteoporosis is inversely associated with arterial stiffness in the elderly: an investigation using the Osteoporosis Self-assessment Tool for Asians index in an elderly Chinese cohort. 2019:21(3):405-11.

47. Nielson CM, Srikanth $\mathrm{P}$, Orwoll ES. Obesity and fracture in men and women: an epidemiologic perspective. Journal of bone and mineral research : the official journal of the American Society for Bone and Mineral Research. 2012; 27(1):1-10.

48. Compston J. Obesity and bone. Current osteoporosis reports. 2013;11(1):30-5.

49. Cui R, Zhou L, Li Z, Li Q, Qi Z, Zhang J. Assessment risk of osteoporosis in Chinese people: relationship among body mass index, serum lipid profiles, blood glucose, and bone mineral density. Clinical interventions in aging. 2016:11:887-95.

50. Chen H, Shao Z, Gao Y, Yu X, Huang S, Zeng P. Are blood lipids risk factors for fracture? Integrative evidence from instrumental variable causal inference and mediation analysis using genetic data. Bone. 2020;131:115174

51. Barngkgei I, Halboub E, Almashraqi AA, Khattab R, Al HI. IDIOS: An innovative index for evaluating dental imaging-based osteoporosis screening indices. Imaging Sci Dent. 2016:46(3):185-202.

\section{Publisher's Note}

Springer Nature remains neutral with regard to jurisdictional claims in published maps and institutional affiliations.

Ready to submit your research? Choose BMC and benefit from:

- fast, convenient online submission

- thorough peer review by experienced researchers in your field

- rapid publication on acceptance

- support for research data, including large and complex data types

- gold Open Access which fosters wider collaboration and increased citations

- maximum visibility for your research: over $100 \mathrm{M}$ website views per year

At BMC, research is always in progress.

Learn more biomedcentral.com/submission 